\title{
On some power series with algebraic coefficients and Liouville numbers
}

\section{Gül Karadeniz Gözeri*}

\section{"Correspondence:}

gulkaradeniz@gmail.com

Department of Mathematics,

Faculty of Science, Istanbul

University, Vezneciler, Istanbul,

Turkiye

\begin{abstract}
In this work, we consider some power series with algebraic coefficients from a certain algebraic number field $K$ of degree $m$ and investigate transcendence of the values of the given series for some Liouville number arguments.
\end{abstract}

\section{Introduction}

The theory of transcendental numbers has a long history and was originated back to Liouville in his famous paper [1] in which he produced the first explicit examples of transcendental numbers at a time where their existence was not yet known. Later, Cantor [2] gave another proof of the existence of transcendental numbers by establishing the denumerability of the set of algebraic numbers. It follows from this that almost all real numbers are transcendental. Further, the theory of transcendental numbers is closely related to the study of Diophantine approximation. Recent advances in Diophantine approximation can be found in the excellent surveys of Moshchevitin [3] and Waldschimidt [4].

Mahler [5] introduced a classification of the set of all transcendental numbers into three disjoint classes, termed $S, T$ and $U$ and this classification has proved to be of considerable value in the general development of the subject. The first classification of this kind was outlined by Maillet in [6], and others were described by Perna in [7] and MorduchaiBoltovskoj [8] but to Mahler's classification attaches by for the most interest. Mahler described this classification in the following way.

Let $P(x)=a_{n} x^{n}+\cdots+a_{1} x+a_{0}$ be a polynomial with integral coefficients. The height $H(P)$ of $P$ is defined by $H(P)=\max \left(\left|a_{n}\right|, \ldots,\left|a_{0}\right|\right)$ and the degree of $P$ is denoted by $\operatorname{deg}(P)$. Given an arbitrary complex number $\xi$, Mahler puts

$$
\omega_{n}(H, \xi)=\min \{|P(\xi)|: \operatorname{deg}(P) \leq n, H(P) \leq H, P(\xi) \neq 0\},
$$

where $n$ and $H$ are positive integers. Next Mahler puts

$$
\omega_{n}(\xi)=\varlimsup_{H \rightarrow \infty} \frac{-\log \omega_{n}(H, \xi)}{\log H} \text { and } \omega(\xi)=\varlimsup_{n \rightarrow \infty} \frac{\omega_{n}(\xi)}{n} \text {. }
$$

The inequalities $0 \leq \omega_{n}(\xi) \leq \infty$ and $0 \leq \omega(\xi) \leq \infty$ hold. From $\omega_{n+1}(H, \xi) \leq \omega_{n}(H$, $\xi)$, we get $\omega_{n+1}(\xi) \geq \omega_{n}(\xi)$. If for an index $\omega_{n}(\xi)=\infty$, the $\mu(\xi)$ is defined as the smallest of them, otherwise $\mu(\xi)=\infty$. Thus, $\mu(\xi)$ is uniquely determined. Furthermore, the two quantities $\mu(\xi)$ and $\omega(\xi)$ are never finite simultaneously, for the finiteness of $\mu(\xi)$ implies that there

\section{空 Springer}

(c) 2013 Karadeniz Gözeri; licensee Springer. This is an Open Access article distributed under the terms of the Creative Commons Attribution License (http://creativecommons.org/licenses/by/2.0), which permits unrestricted use, distribution, and reproduction in any medium, provided the original work is properly cited. 
is an $n<\infty$ such that $\omega_{n}=\infty$, whence $\omega=\infty$. Therefore, there are the following four possibilities for $\xi$, $\xi$ is called

$$
\begin{aligned}
& \text { an } A \text { - number if } \omega(\xi)=0, \mu(\xi)=\infty, \\
& \text { an } S \text { - number if } 0<\omega(\xi)<\infty, \mu(\xi)=\infty, \\
& \text { a } T \text { - number if } \omega(\xi)=\infty, \mu(\xi)=\infty, \\
& \text { a } U \text { - number if } \omega(\xi)=\infty, \mu(\xi)<\infty .
\end{aligned}
$$

In [9], Koksma introduced an analogous classification of complex numbers. He divided the complex numbers into four classes $A^{*}, S^{*}, T^{*}$ and $U^{*}$ in the following way.

Let $\alpha$ be an arbitrary algebraic number. If we denote its minimal defining polynomial by $P(x)$, then the height $H(\alpha)$ of $\alpha$ is defined by $H(\alpha)=H(P)$ and the degree $\operatorname{deg}(\alpha)$ of $\alpha$ is defined by $\operatorname{deg}(\alpha)=\operatorname{deg}(P)$. Given an arbitrary complex number $\xi$ and positive integers $n$, $H$, let $\alpha$ be an algebraic number with degree at most $n$ and height at most $H$ such that $|\xi-\alpha|$ takes the smallest positive value; Koksma defines $\omega_{n}^{*}(H, \xi)$ by the following equation:

$$
\omega_{n}^{*}(H, \xi)=\min \{|\xi-\alpha|: \alpha \text { is algebraic, } \operatorname{deg}(\alpha) \leq n, H(\alpha) \leq H, \alpha \neq \xi\} .
$$

Next, Koksma puts

$$
\omega_{n}^{*}(\xi)=\varlimsup_{H \rightarrow \infty} \frac{-\log \left(H \omega_{n}^{*}(H, \xi)\right)}{\log H} \text { and } \quad \omega^{*}(\xi)=\varlimsup_{n \rightarrow \infty} \frac{\omega_{n}^{*}(\xi)}{n} .
$$

The inequalities $0 \leq \omega_{n}^{*}(\xi) \leq \infty$ and $0 \leq \omega^{*}(\xi) \leq \infty$ hold. If for an index $\omega_{n}^{*}(\xi)=\infty$, the $\mu^{*}(\xi)$ is defined as the smallest of them, otherwise $\mu^{*}(\xi)=\infty$. Thus, $\mu^{*}(\xi)$ is uniquely determined. Furthermore, the two quantities $\mu^{*}(\xi)$ and $\omega^{*}(\xi)$ are never finite simultaneously. Therefore, there are the following four possibilities for $\xi$, $\xi$ is called:

$$
\begin{aligned}
& \text { an } A^{*} \text { - number if } \omega^{*}(\xi)=0, \mu^{*}(\xi)=\infty, \\
& \text { an } S^{*} \text { - number if } 0<\omega^{*}(\xi)<\infty, \mu^{*}(\xi)=\infty, \\
& \text { a } T^{*} \text { - number if } \omega^{*}(\xi)=\infty, \mu^{*}(\xi)=\infty, \\
& \text { a } U^{*} \text { - number if } \omega^{*}(\xi)=\infty, \mu^{*}(\xi)<\infty .
\end{aligned}
$$

Wirsing [10] proved that both classifications are equivalent. Namely, $A, S, T$ and $U$ numbers are the same as $A^{*}, S^{*}, T^{*}$ and $U^{*}$ numbers. The class $A$ is precisely the set of algebraic numbers. $\xi$ is called a $U$-number of degree $m$ if $\mu(\xi)=m$. The set of $U$-numbers of degree $m$ is denoted by $U_{m}$. It is obvious that for any $m \geq 1$, the $U_{m}$ is a subclass of $U$ and $U$ is the union of all disjoint sets $U_{m}$. Leveque [11] proved that $U_{m}$ is not empty for any $m \geq 1$.

In [12], Oryan considered a class of power series with algebraic coefficients and proved that under certain conditions these series take values in the subclass $U_{m}$ for algebraic arguments. Later in [13], similar relations are investigated for Liouville number arguments, and it is proved that these series take values in the set of Mahler's $U$-numbers. In [14], Saradha and Tijdeman considered certain convergent sums and showed that they are either rational or transcendental. Later in [15], Yuan and Li obtained further results for some convergent 
sums. In [16], Nyblom employed a variation on the proof used to established Liouville's theorem concerning the rational approximation of algebraic numbers, to deduce explicit growth conditions for a certain series to converge to a transcendental number. Later, $\mathrm{Ny}$ blom [17] derived a sufficiency condition for a series of positive rational terms to converge to a transcendental number. Further, Duverney [18] proved a theorem that gives a criterion for the sums of infinite series to be transcendental. The terms of these series consist of the rational numbers and converge regularly and very quickly to zero. In [19], Hančl introduced the concept of transcendental sequences and proved a criterion for sequences to be transcendental. Later, a new concept of a Liouville sequence was introduced in [20] by means of the related Liouville series. Some recent results for the transcendence of infinite series can also be found in Borwein and Coons [21], Hančl and Rucki [22], Hančl and Štěpnička [23], Murty and Weatherby [24], Weatherby [25].

In the present work, we considered certain power series with algebraic coefficients from a certain algebraic number field $K$ of degree $m$ and showed that under certain conditions these series take values belonging to either the algebraic number field $K$ or $\bigcup_{i=1}^{m} U_{i}$ in Mahler's classification of the complex numbers for some Liouville number arguments.

\section{Preliminaries}

In this paper, $|x|$ means the absolute value of $x$ and the least common multiple of $x_{1}, x_{2}, \ldots, x_{n}$ is denoted by $\left[x_{1}, x_{2}, \ldots, x_{n}\right]$.

Definition 1 A real number $\xi$ is called a Liouville number if and only if for every positive integer $n$ there exists integers $p_{n}, q_{n}\left(q_{n}>1\right)$ with

$$
0<\left|\xi-\frac{p_{n}}{q_{n}}\right|<\frac{1}{q_{n}^{n}} .
$$

The set of all Liouville numbers is identical with the $U_{1}$ subclass. More information about Liouville numbers may be found in [26-28]. Now, in order to prove our main theorem we need the following lemmas.

Lemma 2 [29] Let $\alpha_{1}, \ldots, \alpha_{k}(k \geq 1)$ be algebraic numbers which belong to an algebraic number field $K$ of degree $m$, and let $F\left(y, x_{1}, \ldots, x_{k}\right)$ be a polynomial with rational integral coefficients and with degree at least 1 in $y$. If $\eta$ is any algebraic number such that $F\left(\eta, \alpha_{1}, \ldots, \alpha_{k}\right)=0$, then $\operatorname{deg}(\eta) \leq d m$ and

$$
H(\eta) \leq 3^{2 d m+\left(l_{1}+\cdots+l_{k}\right) m} H^{m} H\left(\alpha_{1}\right)^{l_{1} m} \cdots H\left(\alpha_{k}\right)^{l_{k} m},
$$

where $H$ is the height of the polynomial $F, d$ is the degree of $F$ in $y$ and $l_{i}$ is the degree of $F$ in $x_{i}$ for $i=1, \ldots, k$.

Lemma 3 [11] Let $\alpha$ be an algebraic number of degree $m$, and let $\alpha^{(1)}=\alpha, \ldots, \alpha^{(m)}$ be its conjugates. Then $\overline{|\alpha|} \leq 2 H(\alpha)$, where $\overline{|\alpha|}=\max \left(\left|\alpha^{(1)}\right|, \ldots,\left|\alpha^{(m)}\right|\right)$.

Lemma 4 [30] Let $\alpha$ be an algebraic number of degree $m$, then $H(\alpha) \leq(2 \overline{|\alpha|})^{m}$, where $\overline{|\alpha|}=\max \left(\left|\alpha^{(1)}\right|, \ldots,\left|\alpha^{(m)}\right|\right)$. 


\section{Main result}

Theorem 5 Let $K$ be an algebraic number field of degree $m$, and let

$$
g(x)=\sum_{n=0}^{\infty} \frac{\alpha_{n}}{e_{n}} x^{n}
$$

be a power series such that $\alpha_{n} \in K$ are non-zero algebraic numbers and $e_{n}>1$ are rational integers satisfying the following conditions:

$$
\begin{aligned}
& \varliminf_{n \rightarrow \infty} \frac{\log e_{n+1}}{\log e_{n}}=\eta>1, \\
& \varlimsup_{n \rightarrow \infty} \frac{\log e_{n+1}}{\log e_{n}}=\infty, \\
& \varlimsup_{n \rightarrow \infty} \frac{\log H\left(\alpha_{n}\right)}{\log e_{n}}=\mu<1 .
\end{aligned}
$$

Further, let $\xi$ be a Liouville number satisfying the following two properties:

1. $\xi$ has an approximation with rational numbers $\frac{p_{n}}{q_{n}}\left(q_{n}>1\right)$ so that the following inequality holds for sufficiently large $n$

$$
\left|\xi-\frac{p_{n}}{q_{n}}\right|<\frac{1}{q_{n}^{n s_{n}}} \quad\left(\lim _{n \rightarrow \infty} s_{n}=+\infty\right) .
$$

2. There exist two positive real constants $\gamma_{1}$ and $\gamma_{2}$ with $\frac{\eta}{\eta-1}<\gamma_{1}<\gamma_{2}$ and

$$
e_{n}^{\gamma_{1}} \leq q_{n}^{n} \leq e_{n}^{\gamma_{2}}
$$

for sufficiently large $n$.

Then $g(\xi)$ belongs to either the algebraic number field $K$ or $\bigcup_{i=1}^{m} U_{i}$.

Proof It follows from (1) that

$$
\log e_{n+1}>\eta_{1} \log e_{n}
$$

for sufficiently large $n$, where $\eta_{1}=\eta-\varepsilon_{1}$ and $\varepsilon_{1}$ is to be chosen as $0<\varepsilon_{1}<\eta-\frac{\gamma_{1}}{\gamma_{1}-1}$. It follows from (6) that the sequence $\left\{e_{n}\right\}$ is strictly increasing, thus we have

$$
\begin{aligned}
& \lim _{n \rightarrow \infty} e_{n}=\infty, \\
& \lim _{n \rightarrow \infty} \frac{\log e_{n}}{n}=\infty \text { and } \lim _{n \rightarrow \infty} \frac{\log e_{n}}{n^{2}}=\infty
\end{aligned}
$$

Furthermore, from (6) we get

$$
e_{n}<e_{n+1}^{\frac{1}{\eta_{1}}}
$$

for sufficiently large $n$. 
Let $E_{n}=\left[e_{0}, e_{1}, \ldots, e_{n}\right]$. Then by using (7) and (9), we obtain for sufficiently large $n$

$$
e_{n} \leq E_{n} \leq e_{n}^{\varepsilon_{2}+\frac{\eta_{1}}{\eta_{1}-1}}
$$

where $\varepsilon_{2}$ is to be chosen as $0<\varepsilon_{2}<\gamma_{1}-\frac{\eta_{1}}{\eta_{1}-1}$. We can easily deduce from (3) and $\mu<\frac{\mu+1}{2}$ that

$$
\frac{\log H\left(\alpha_{n}\right)}{\log e_{n}}<\frac{\mu+1}{2}
$$

for sufficiently large $n$. Since (11) holds, there is a natural number $N_{0}$ such that

$$
H\left(\alpha_{n}\right)<e_{n}^{\left(\frac{\mu+1}{2}\right)}
$$

for every $n>N_{0}$. On the other hand, we get from Lemma 3 that

$$
\overline{\left|\alpha_{n}\right|} \leq 2 H\left(\alpha_{n}\right)
$$

since $\alpha_{n}$ are algebraic numbers. From here and (12), we obtain

$$
\left|\alpha_{n}\right| \leq \overline{\left|\alpha_{n}\right|} \leq 2 e_{n}^{\left(\frac{\mu+1}{2}\right)}
$$

for every $n>N_{0}$. Now, we shall define the algebraic numbers

$$
\beta_{n}=\sum_{\gamma=0}^{n} \frac{\alpha_{\gamma}}{e_{\gamma}}\left(\frac{p_{n}}{q_{n}}\right)^{\gamma}
$$

for $n=0,1,2, \ldots$. Since $\beta_{n} \in K, \operatorname{deg}\left(\beta_{n}\right) \leq m$ for $n=0,1,2, \ldots$. Let us determine an upper bound for the heights of the algebraic numbers $\beta_{n}$. By multiplying both sides of this equality by $E_{n} q_{n}^{n}$ and putting $l_{i}=\frac{E_{n}}{e_{i}}$ for $i=0,1,2, \ldots$, we obtain the equality

$$
E_{n} q_{n}^{n} \beta_{n}=\left(l_{0} q_{n}^{n}\right) \alpha_{0}+\left(l_{1} q_{n}^{n-1} p_{n}\right) \alpha_{1}+\cdots+\left(l_{n} p_{n}^{n}\right) \alpha_{n} .
$$

Since $\xi$ is a Liouville number, we can assume that $p_{n} \neq 0$ for $n=0,1,2, \ldots$. Then we get a polynomial

$$
G\left(y, x_{0}, x_{1}, \ldots, x_{n}\right)=E_{n} q_{n}^{n} y-\sum_{\gamma=0}^{n}\left(l_{\gamma} q_{n}^{n-\gamma} p_{n}^{\gamma}\right) x_{\gamma}
$$

with rational integral coefficients such that $G\left(\beta_{n}, \alpha_{0}, \ldots, \alpha_{n}\right)=0$. Further, this polynomial is of degree 1 in each $y, x_{0}, x_{1}, \ldots, x_{n}$. Thus, we deduce from Lemma 2 that

$$
H\left(\beta_{n}\right) \leq 3^{2 m+(n+1) m} H^{m} H\left(\alpha_{0}\right)^{m} \cdots H\left(\alpha_{n}\right)^{m},
$$

where $H$ is the height of the polynomial $G\left(y, x_{0}, x_{1}, \ldots, x_{n}\right)$. By using (4), we obtain $\left|\frac{p_{n}}{q_{n}}\right|^{i} \leq$ $k_{0}^{n}$ for $i=0,1,2, \ldots$, where $k_{0}=|\xi|+1>1$ is a real constant. From here, we can easily get

$$
H \leq E_{n} q_{n}^{n} k_{0}^{n}
$$


It follows from (15) and (16) that

$$
H\left(\beta_{n}\right) \leq k_{1}^{m n} E_{n}^{m} q_{n}^{m n} H\left(\alpha_{0}\right)^{m} \cdots H\left(\alpha_{n}\right)^{m},
$$

where $k_{1}=3{ }^{4} k_{0}>1$ is a real constant. Moreover, we get

$$
H\left(\beta_{i}\right) \leq\left(2 \mid \overline{\beta_{i} \mid}\right)^{m} \quad(i=0,1,2, \ldots)
$$

from Lemma 4. Then we deduce from (17) and (18) that

$$
H\left(\beta_{n}\right) \leq 2^{m^{2}(n+1)} k_{1}^{m n} E_{n}^{m} q_{n}^{m n}\left(\overline{\left|\beta_{0}\right|} \cdots \overline{\left|\beta_{n}\right|}\right)^{m^{2}} .
$$

By using (10), (13) and (14), we obtain

$$
\left(\overline{\left|\beta_{0}\right|} \cdots \overline{\left|\beta_{n}\right|}\right)^{m^{2}} \leq 2^{m^{2}(n+1)} k_{2} e_{n}^{\gamma_{1} m^{2}\left(\frac{\mu+1}{2}\right)}
$$

where $k_{2}=\max \left(1,\left(H\left(\alpha_{0}\right) \cdots H\left(\alpha_{N_{0}}\right)\right)^{m^{2}}\right) \geq 1$ is a real constant. It follows from here, (5) and (10) that

$$
H\left(\beta_{n}\right) \leq k_{3}^{n} e_{n}^{k_{4}}
$$

where $k_{3}=2^{4 m^{2}} k_{1}^{m} k_{2}>1$ and $k_{4}=\gamma_{1} m+\gamma_{1} m^{2}\left(\frac{\mu+1}{2}\right)+\gamma_{2} m>1$ are real constants.

Now, we consider the following polynomials:

$$
g_{n}(x)=\sum_{v=0}^{n} \frac{\alpha_{v}}{e_{v}} x^{v}
$$

for $n=1,2, \ldots$. Since $g_{n}(x)$ are continuous and differentiable for all real numbers, at least one real number $c_{n}$ exists between $\xi$ and $\frac{p_{n}}{q_{n}}$ such that for every $n$

$$
g_{n}(\xi)-\beta_{n}=g_{n}(\xi)-g_{n}\left(\frac{p_{n}}{q_{n}}\right)=\left(\xi-\frac{p_{n}}{q_{n}}\right) g_{n}^{\prime}\left(c_{n}\right) .
$$

It is obvious that $\left|c_{n}\right| \leq \max \left(|\xi|,\left|\frac{p_{n}}{q_{n}}\right|\right)$. Since $\left|\frac{p_{n}}{q_{n}}\right|<|\xi|+1$, we obtain $\left|c_{n}\right| \leq|\xi|+1$ for sufficiently large $n$. Furthermore, from here and (4) and (21), we get

$$
\left|g_{n}(\xi)-g_{n}\left(\frac{p_{n}}{q_{n}}\right)\right| \leq \frac{1}{q_{n}^{n s_{n}}} \sum_{\nu=1}^{n} \frac{\left|\alpha_{v}\right|}{e_{v}} v(|\xi|+1)^{\nu-1}
$$

for sufficiently large $n$.

Define $\sigma_{n}=\max \left(\left|\alpha_{0}\right|, \ldots,\left|\alpha_{n}\right|\right)$. Then we obtain

$$
\sum_{\nu=1}^{n} \frac{\left|\alpha_{\nu}\right|}{e_{\nu}} v(|\xi|+1)^{\nu-1} \leq n^{2} \sigma_{n}(|\xi|+1)^{n-1}
$$


for sufficiently large $n$. It follows from (14) that $\sigma_{n} \leq 2 e_{n}^{\left(\frac{1+\mu}{2}\right)}$ for sufficiently large $n$. We get from here and (22), (23)

$$
\left|g_{n}(\xi)-\beta_{n}\right| \leq \frac{2 n^{2}(|\xi|+1)^{n-1} e_{n}^{\left(\frac{1+\mu}{2}\right)}}{q_{n}^{n s_{n}}}
$$

By using (5), we obtain from here that

$$
\left|g_{n}(\xi)-\beta_{n}\right| \leq \frac{2 n^{2}(|\xi|+1)^{n-1}}{e_{n}^{\gamma_{1} s_{n}-\left(\frac{1+\mu}{2}\right)}}
$$

From (8) and $\lim _{n \rightarrow \infty} s_{n}=\infty$, it is possible to find a sequence $\left\{\omega_{n}^{\prime}\right\}$ with $\lim _{n \rightarrow \infty} \omega_{n}^{\prime}=\infty$ such that

$$
\frac{2 n^{2}(|\xi|+1)^{n-1}}{e_{n}^{\gamma_{1} s_{n}-\left(\frac{1+\mu}{2}\right)}} \leq \frac{1}{2\left(k_{3}^{n} e_{n}^{k_{4}}\right)^{\omega_{n}^{\prime}}}
$$

Therefore, we get from (20), (24) and (25)

$$
\left|g_{n}(\xi)-\beta_{n}\right| \leq \frac{1}{2 H\left(\beta_{n}\right)^{\prime \prime}}
$$

for sufficiently large $n$. Moreover, the following inequality holds:

$$
\left|g(\xi)-g_{n}(\xi)\right| \leq \sum_{i=1}^{\infty} \frac{\left|\alpha_{n+i}\right|}{e_{n+i}}|\xi|^{n+i}
$$

We get from here and (14)

$$
\left|g(\xi)-g_{n}(\xi)\right| \leq \frac{2|\xi|^{n+1}}{e_{n+1}^{\left(\frac{1-\mu}{2}\right)}}\left[1+\left(\frac{e_{n+1}}{e_{n+2}}\right)^{\frac{1-\mu}{2}}|\xi|+\left(\frac{e_{n+1}}{e_{n+3}}\right)^{\frac{1-\mu}{2}}|\xi|^{2}+\cdots\right] .
$$

Thus, we deduce from (9) that

$$
0<\frac{e_{n+1}}{e_{n+2}}<\frac{1}{e_{n+2}^{\left(1-\frac{1}{\eta_{1}}\right)}} .
$$

Since (7) holds, then we obtain $\lim _{n \rightarrow \infty} \frac{e_{n+1}}{e_{n+2}}=0$. Similarly, since $\frac{1-\mu}{2}>0$, we have

$$
\left(\frac{e_{n+1}}{e_{n+1+k}}\right)^{\frac{1-\mu}{2}}|\xi|^{k}<\left(\frac{1}{2}\right)^{k}
$$

for $k=1,2,3, \ldots$ and, therefore,

$$
\left|g(\xi)-g_{n}(\xi)\right| \leq \frac{4|\xi|^{n+1}}{e_{n+1}^{\left(\frac{1-\mu}{2}\right)}}
$$


On the other hand from (8), we get $4|\xi|^{n+1} \leq e_{n+1}^{\left(\frac{1-\mu}{4}\right)}$ for sufficiently large $n$. From here, we obtain

$$
\left|g(\xi)-g_{n}(\xi)\right| \leq \frac{1}{e_{n+1}^{\left(\frac{1-\mu}{4}\right)}}
$$

for sufficiently large $n$. Now if we define $r_{n}=\frac{\log e_{n+1}}{\log e_{n}}$, then we have

$$
\left|g(\xi)-g_{n}(\xi)\right| \leq \frac{1}{e_{n}^{r_{n}\left(\frac{1-\mu}{4}\right)}}
$$

Using (2), then it follows that there exists a subsequence $\left\{r_{n_{k}}\right\}$ of $\left\{r_{n}\right\}$ such that $\lim _{k \rightarrow \infty} r_{n_{k}}=$ $\infty$. Therefore, we get

$$
\left|g(\xi)-g_{n_{k}}(\xi)\right| \leq \frac{1}{e_{n_{k}}^{r_{n_{k}}\left(\frac{1-\mu}{4}\right)}}
$$

for sufficiently large $n_{k}$. From $(8)$ and $\lim _{k \rightarrow \infty} r_{n_{k}}=\infty$, there exists a suitable sequence $\left\{r_{n_{k}}^{\prime}\right\}$ with $\lim _{k \rightarrow \infty} r_{n_{k}}^{\prime}=\infty$ such that

$$
\frac{1}{e_{n_{k}}^{r_{n_{k}}\left(\frac{1-\mu}{4}\right)}} \leq \frac{1}{2\left(k_{3}^{n} e_{n}^{k_{4}}\right)^{r_{n_{k}}^{\prime}}}
$$

and, therefore, from (20) and (27), we obtain

$$
\left|g(\xi)-g_{n_{k}}(\xi)\right| \leq \frac{1}{2 H\left(\beta_{n_{k}}\right)^{r_{n_{k}}}}
$$

for sufficiently large $n_{k}$. On the other hand by using (26), we deduce that

$$
\left|g_{n_{k}}(\xi)-\beta_{n_{k}}\right| \leq \frac{1}{2 H\left(\beta_{n_{k}}\right)^{\omega_{n_{k}}^{\prime}}}
$$

for sufficiently large $n_{k}$. Let $\omega_{n_{k}}=\min \left(r_{n_{k}}^{\prime}, \omega_{n_{k}}^{\prime}\right)$. It follows from (28) and (29) that

$$
\left|g(\xi)-\beta_{n_{k}}\right| \leq \frac{1}{H\left(\beta_{n_{k}}\right)^{\omega_{n_{k}}}}
$$

where $\lim _{k \rightarrow \infty} \omega_{n_{k}}=\infty$. It follows from here that $\lim _{k \rightarrow \infty} \beta_{n_{k}}=g(\xi)$. Thus, if the sequence $\left\{\beta_{n_{k}}\right\}$ is constant, then $g(\xi)$ is an algebraic number in $K$. Otherwise $g(\xi) \in \bigcup_{i=1}^{m} U_{i}$.

\section{Conclusion}

In this work, the series with algebraic coefficients are treated and it is shown that under certain conditions the values of these series are either algebraic numbers or $U$-numbers for Liouville number arguments. The similar results can be proved for the power series, which are defined in the $p$-adic field $Q_{p}$ and in the field of formal Laurent series. 


\section{Competing interests}

The author declares that she has no competing interests.

\section{Acknowledgements}

Dedicated to Professor Hari M Srivastava.

The author would like to express her sincere thanks to the referees for their careful reading and for making some valuable comments, which have essentially improved the presentation of this paper.

\section{Received: 14 December 2012 Accepted: 2 April 2013 Published: 16 April 2013}

\section{References}

1. Liouville, J: Sur des classes trés étendues de quantités dont la valeur n'est ni algébrique, ni méme reductible à des irrationnelles algébriques. C. R. Acad. Sci. 18, 883-885 (1844)

2. Cantor, G: Über eine Eigenschaft des Inbegriffs aller reellen algebraishen Zahlen. J. Reine Angew. Math. 77, 258-262 (1874)

3. Moshchevitin, NG: Khintchine's singular Diophantine systems and their applications. Usp. Mat. Nauk 65, 43-126 (2010)

4. Waldschmidt, M: Recent advances in Diophantine approximation. In: Number Theory, Analysis and Geometry: In Memory of Serge Lang, pp. 659-704. Springer, New York (2012)

5. Mahler, K: Zur Approximation der Exponentialfunktion und des Logarithmus I, II. J. Reine Angew. Math. 166, 118-150 (1932)

6. Maillet, E: Sur la classification des irrationelles. C. R. Acad. Sci. 143, 26-28 (1906)

7. Perna, A: Sui numeri transcendenti in generale e sulla loroconstruzione in base al criterio di Liouville. G. Mat. Battaglini 52, 305-365 (1914)

8. Morduchai-Boltovskoj, D: On transcendental numbers with successive approximations defined by algebraic equations. Rec. Math. Moscou 41, 221-232 (1934)

9. Koksma, JF: Über die Mahlersche Klasseneinteilung der transzendenten Zahlen und die Approximation komplexer Zahlen durch algebraische Zahlen. Monatshefte Math. Phys. 48, 176-189 (1939)

10. Wirsing, E: Approximation mit algebraischen Zahlen beschränkten Grades. J. Reine Angew. Math. 206, 67-77 (1961)

11. Leveque, WJ: On Mahler's U-numbers. J. Lond. Math. Soc. 28, 220-229 (1953)

12. Oryan, MH: Über gewisse Potenzreihen, die für algebraische Argumente Werte aus Der Mahlerschen Unterklassen $U_{m}$ nehmen. Istanbul Univ. Fen Fak. Mecm., Seri A 45, 1-42 (1980)

13. Oryan, MH: Über gewisse Potenzreihen, deren Funktionswerte für Argumente aus der Menge der Liouvilleschen Zahlen U-Zahlen vom Grade $\leq m$ sind. Istanbul Univ. Fen Fak. Mecm., Seri A 47, 15-34 (1983-1986)

14. Saradha, N, Tijdeman, R: On the transcendence of some infinite sums of values of rational functions. J. Lond. Math. Soc. 67(3), 580-592 (2003)

15. Yuan, P, Li, J: On the transcendence of some infinite sums. J. Lond. Math. Soc. 80(2), 431-445 (2009)

16. Nyblom, MA: A theorem on transcendence of infinite series. Rocky Mt. J. Math. 30, 1-10 (2000)

17. Nyblom, MA: A theorem on transcendence of infinite series II. J. Number Theory 91, 71-80 (2001)

18. Duverney, D: Transcendence of a fast converging series of rational numbers. Math. Proc. Camb. Philos. Soc. 130 193-207 (2001)

19. Hančl, J: Two criteria for transcendental sequences. Matematiche 56, 129-140 (2002)

20. Hančl, J: Liouville sequences. Nagoya Math. J. 172, 173-187 (2003)

21. Borwein, $\mathrm{P}$, Coons, M: Transcendence of power series for some number theoretic functions. Proc. Am. Math. Soc. 137, 1303-1305 (2009)

22. Hančl, J, Rucki, P: Certain Liouville series. Ann. Univ. Ferrara, Sez. 7: Sci. Mat. 52, 45-51 (2006)

23. Hančl, J, Štěpnička, J: On the transcendence of some infinite series. Glasg. Math. J. 50, 33-37 (2008)

24. Murty, MR, Weatherby, CJ: On the transcendence of certain infinite series. Int. J. Number Theory 7(2), 323-339 (2011)

25. Weatherby, CJ: Transcendence of multi-indexed infinite series. J. Number Theory 131(4), 705-715 (2011)

26. Burger, EB, Tubbs, R: Making Transcendence Transparent. Springer, New York (2004)

27. Leveque, WJ: Topics in Number Theory, vol. 2. Addison-Wesley, London (1956)

28. Perron, O: Irrationalzahlen. de Gruyter, Berlin (1960)

29. '̇çen, OŞ: Anhang zu den Arbeiten 'Über die Funktionswerte der $p$-adischen elliptischen Funktionen I und II'. Istanbul Univ. Fen Fak. Mecm., Seri A 38, 25-35 (1973)

30. Schneider, T: Einführung in die transzendenten Zahlen. Springer, Berlin (1957)

doi:10.1186/1029-242X-2013-178

Cite this article as: Karadeniz Gözeri: On some power series with algebraic coefficients and Liouville numbers.

Journal of Inequalities and Applications 2013 2013:178. 